DOI:

$10.1038 /$ nrm2333

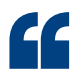

This differential requirement

for nuclear export factors is likely to signify the increase in complexity in metazoan genomes...
$\Rightarrow$ NUCLEAR TRANSPORT

\section{Exit for fly mRNA}

Pamela Silver and colleagues report the first genome-wide RNA interference (RNAi) screen for factors that are required for the nuclear export of mRNAs in a metazoan system.

The visual screen, which was based on the subcellular localization of mRNA in RNAi-treated cells (cells that are defective in a nuclear export factor accumulate mRNA in the nucleus), led to the identification of 72 factors that are required for mRNA nuclear export in Drosophila melanogaster. Of these, 18 were required for $\mathrm{mRNA}$ export in both yeast and flies, whereas the 54 additional factors could be categorized as having functions in RNA processing, translation, the cell cycle or unknown processes.

By comparing the nuclear export requirement of two heat-shockinducible transcripts from flies - the intronless HSP70 mRNA and the intron-containing HSP83 mRNA - the authors found that those export factors that were necessary for HSP70 mRNA export were also required for HSP83 mRNA export. Conversely, however, HSP83 mRNA required additional export factors, which might reflect the extra mRNA processing requirements for introncontaining genes. This differential requirement for nuclear export factors is likely to signify the increase in complexity in metazoan genomes compared with that of yeast.

Among the newly identified nuclear export factors, several were found to interact with the core mRNA export machinery or were part of a compensatory transcriptional network that was able to upregulate core export factors in response to an export blockage. Whereas these novel factors need to be characterized in more detail, some are likely to be direct participants in mRNA export.

The identification of other new factors revealed connections between mRNA export and both translation and cell-cycle regulation. One such factor, dmPCID2, associates with actively translating ribosomes, and this interaction depends on the presence of a PCI domain (a protein-protein interaction domain that is also present in eIF3 translation initiation factors). The authors speculate that dmPCID2 may function as a bridge between mRNA export and translation, possibly through the interaction between the export machinery and the PCI-containing eIF3 complex.

This screen provides a useful foundation for analysing mRNA export in yeast and higher eukaryotes, and for characterizing new factors and exploring new links between the processes - from mRNA transcription, processing and export to translation - that regulate gene expression.

\section{Arianne Heinrichs}

ORIGINAL RESEARCH PAPER Farny, N. G., Hurt, J. A. \& Silver, P. A. Definition of global and transcript-specific mRNA export pathways in metazoans. Genes Dev. 17 Dec 2007 (doi:10.1101/gad.1616008) FURTHER READING Kohler, A. \& Hurt, E. Exporting RNA from the nucleus to the cytoplasm. Nature Rev. Mol. Cell Biol. 8, 761-773 (2007) 